\title{
Electron Exit Wave Reconstruction From a Single Defocused Image Using a Gaussian Basis
}

\author{
Konstantin B. Borisenko, Christopher S. Allen, Jamie H. Warner and Angus I. Kirkland \\ Department of Materials, University of Oxford, Parks Road, Oxford, OX1 3PH, UK
}

Aberration-corrected high resolution transmission electron microscopy (HRTEM) is a well established tool for studying the atomic structures of materials. Using a series of images collected with different foci it is possible to reconstruct the electron exit wavefunction $[1,2]$.

The accuracy of this type of reconstruction, however, is limited by the ability of the sample to withstand radiation damage during the long acquisition time required for data collection. The usefulness of this method is hence limited for radiation sensitive materials.

The signal to noise ratio in the reconstruction also depends on the number of images recorded in the focal series. Usually, 30 or more images are collected, which provides the necessary redundancy in the experimental data for reliable reconstruction of the exit wave phase and amplitude.

Special steps are therefore required to reduce radiation damage and increase the accuracy of the reconstruction if such a method is to be applied to radiation sensitive materials. One approach is to use a lower radiation dose to record the focal series [3]. This, however, can lead to problems with aberration measurement and alignment of noisy image data.

Another possible approach is to reduce the number of collected experimental data, in principle, down to a single image. However, one of the barriers to successful exit wave reconstruction is that in this case the number of variables in the restored complex exit wave, if each pixel is treated as a variable, is twice as large as the number of the recorded experimental data (number of pixels) in the image. The presence of objective lens aberrations and detector noise further complicates the analysis and can lead to multiple and unstable reconstructions.

In the present work we present a new algorithm for exit wave reconstruction from a single defocused image of a weak-phase object and also a more general object. By representing the electron exit wave in a Gaussian basis it is possible to greatly reduce the number of variables needed to find a solution, in a way that is related to compressed sensing [4]. To illustrate this we compare the reconstructed experimental exit wave phase from graphene and silicon nitride using a Gaussian basis with the corresponding exit wave reconstructed using a conventional linear Wiener filter restoration from an extended focal series.

In a weak-phase object approximation exit wave has a complex amplitude: $W(x, y)=A e^{i \phi(x, y)}$, where $\phi(x, y)$ is phase shift, and $A$ is an amplitude, $A=1$. We represent the exit wave phase as a sum of $n$ symmetric Gaussian functions $G$ as $\phi(x, y)=\sum_{n} G_{n}\left(a_{n}, b_{x n}, b_{y n}, c_{n}, x, y\right)$, where the Gaussian functions in two dimensions have general form $G\left(a, b_{x}, b_{y}, c, x, y\right)=a e^{\frac{-\left(x-b_{x}\right)^{2}-\left(y-b_{y}\right)^{2}}{2 c^{2}}}$, and are described by four independent parameters, defining their position, height and width. 
In case of coherent or partially coherent illumination the image intensity in the real space is obtained by computing the squared modulus of a convolution of the point spread function of the electron microscope $P$ with the electron exit wave, $I(x, y)=|W(x, y) \otimes P(x, y)|^{2}$.

The image intensity is therefore represented as a function of parameters of the Gaussians in the exit wave and the parameters of the point spread function of the microscope, or objective lens aberration function in reciprocal space. With known parameters for the lens aberration function, the parameters of the exit wave can be found by minimising the sum of squared differences between the simulated and experimental real space images, or alternatively, by solving an overdetermined system of non-linear equations, $\left.I\left(x, y, a_{n}, b_{x n}, b_{y n}, c_{n}\right)^{s i m}-I(x, y)^{\exp }=0\right\}$

The reconstructed exit wave phase from a single defocused image of a monolayer graphene sample using the representation in a Gaussian basis is presented in Figure 1. As the exit wave phase is sensitive to the three-dimensional structure of the layer, brighter regions discernible in the exit wave phase may represent out-of-plane ripples around the location of a dislocation dipole, indicated in the image.

\section{References}

[1] WMJ Coene, A Thust, M deBeeck and D VanDyck, Ultramicroscopy 64 (1996), p. 109.

[2] WK Hsieh, FR Chen, JJ Kai and AI Kirkland, Ultramicroscopy 98 (2004), p. 99.

[3] B Barton, B Jiang, C Song, P Specht, H Calderon and C Kisielowski, Microsc. Microanal. 18 (2012), p. 982.

[4] EJ Candès and MB Wakin, IEEE Signal Proc. Mag. 25 (2008), p. 21.

[5] Financial support from the European Union under the Seventh Framework Program under a contract for an Integrated Infrastructure Initiative (Ref. 312483-ESTEEM2) is gratefully acknowledged.

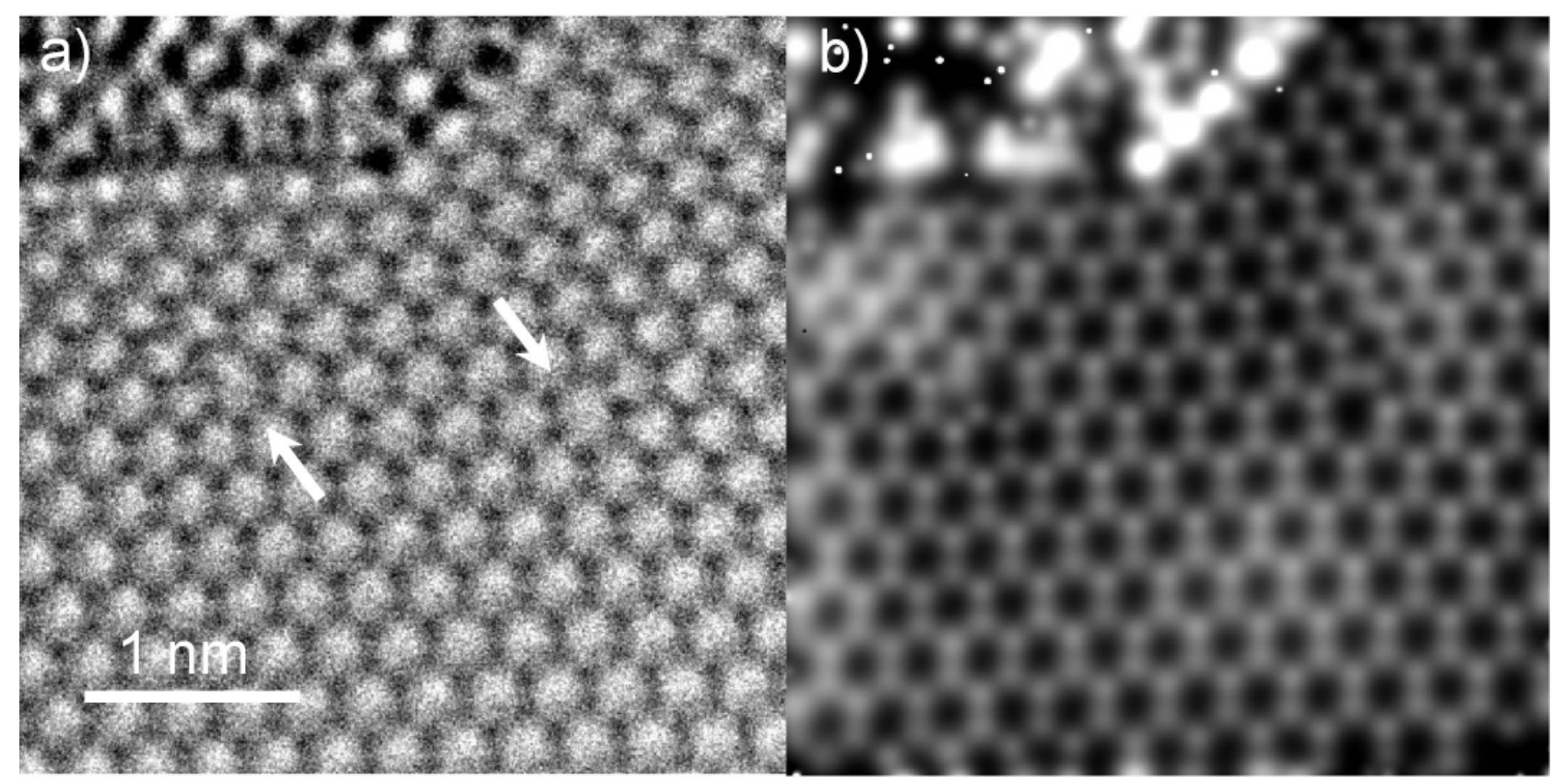

Figure 1. Experimental image of monolayer graphene a) and the reconstructed exit wave phase using representation in a Gaussian basis b). A dislocation dipole is indicated by arrows in the image. 